\title{
A Volterra functional-integral equation, via weakly Picard operators' technique
}

\section{VIORICA MUREŞAN}

\section{ABSTRACT.}

In this paper we consider a Volterra functional-integral equation with linear modifications of the arguments. We use weakly Picard operators' technique and we obtain existence and data dependence results for the solutions.

\section{REFERENCES}

[1] Azbelev, N. V., Maksimov, V. P. and Rahmatulina, L. F., Introduction to Functional - Differential Equations Theory, MIR, Moscow, 1991 (In Russian)

[2] Avramescu, C., Sur l'existence des equations integrales dans certaines espaces fonctionnels, Ann. Univ. Scient. Budapesinensis de Roland Eotvos, 13 (1970), 19-34

[3] Corduneanu, C., Abstract Volterra equation: a survey, Nonlinear Operator Theory, Math. Comput. Modelling, 32 (2000), No.11-13, 1503-1528

[4] Guo, D., Lakshmikantham, V. and Liu, X., Nonlinear Integral Equations in Abstract Spaces, Kluwer Acad. Publ., Dordrecht, 1996

[5] Hale, J. K. and Sjoerd, M. Verduyn Lunel, Introduction to Functional - Differential Equations, Springer Verlag, New York, 1993

[6] Ilea, V. and Otrocol, D., Integro-differential equations with two times modifications, Carpathian J. Math., 27 (2011), No. 2, 209-216

[7] Ilea, V. and Otrocol, D., Şerban, M. A. and Trif, D., Integro-differential equation with two time lags, Fixed Point Theory, 13 (2012), No. 1, 85-99

[8] Iserles, A., On the generalized pantograph functional - differential equation, European J. Appl. Math., 4 (1992), $1-38$

[9] Kato, T. and Mc Leod, J. B., The functional - differential equation $y^{\prime}(x)=a y(\lambda x)+b y(x)$, Bull. Amer. Math. Soc., 77 (1971), No. 6, 891-937

[10] Mureşan, V., Functional - Integral Equations, Mediamira, Cluj - Napoca, 2003

[11] Ockendon, J. R., Differential equations and industry, The Math. Scientist, 5 (1980), No. 1, 1-12

[12] Otrocol, D., Abstract Volterra operators, Carpathian J. Math., 24 (2008), No. 3, 370-377

[13] Precup, R., Methods in Nonlinear Integral Equations, Kluwer Acad. Publ., Dordrecht, 2002

[14] Rus, I. A., Weakly Picard mappings, Comment. Math. Univ. Carolinae, 34 (1993), No. 41, 769-773

[15] Rus, I. A., A class of nonlinear functional-integral equations, via weakly Picard operators, Ann. Univ. Craiova, Ser. Mat.- Infor., 28 (2001), 10-15

[16] Rus, I. A., Picard operators and applications, Scientiae Mathematicae Japonicae, 58 (2003), No. 1, 191-219

[17] Rus, I. A., Some nonlinear functional differential and integral equations, via weakly Picard operator theory: a survey, Carpathian J. Math, 26 (2010), No. 2, 230-258

[18] Rus, I. A., Petruşel, A. and Şerban, M. A., Fibre Picard operators on gauge spaces and applications, Journal for Analysis and its Applications, 27 (2008), No. 4, 399-415

[19] Rus, I. A., Şerban, M. A. and Trif, D., Step method for some integral equations from biomathematics, Bull. Math. Soc. Sci. Math. Roumanie, 54 (102) (2011), No. 2, 167-183

[20] Szep, G., On an integral equation with deviating argument, Seminar on Fixed Point Theory, Cluj-Napoca, 1 (2000), 103-108

Received: 29.10.2013; In revised form: 09.09.2014; Accepted: 20.10.2014

2010 Mathematics Subject Classification. 34K05, 34K15, 47H10.

Key words and phrases. Fixed point, Picard operator, weakly Picard operator, functional-integral equation, data dependence. 
[21] Şerban, M. A., Rus I. A. and Petruşel, A., A class of abstract Volterra equations, via weakly Picard operators' technique, Mathematical Inequalities \& Applications, 13 (2010), No. 2, 255-269

TECHNICAL UNIVERSITY OF CLUJ-NAPOCA

FACUlTY OF AUTOMATION AND CUMPUTER SCIENCE

DEPARTMENT OF MATHEMATICS

Memorandumului 28, 400114 Cluj-Napoca, Romania

E-mail address: vmuresan@math.utcluj.ro 